\title{
A two-miRNA signature (miR-21 and miR-92) in peripheral whole blood as a potential biomarker for diagnosis of human cerebral aneurysms
}

\author{
Congying Zheng, Chengliang Mao, Kai Tang, Shaojian Ceng, Hang Shu
}

Department of Neurosurgery, Guangdong Provincial People's Hospital, Guangdong Academy of Medical Sciences, Guangzhou, China

Submitted: 20 August 2019

Accepted: 12 January 2020

Arch Med Sci

DOI: https://doi.org/10.5114/aoms.2020.93536

Copyright @ 2020 Termedia \& Banach

\section{Abstract}

Introduction: microRNAs (miRs) have been reported as blood-based noninvasive indicators for the diagnosis of various diseases. However, the utility of whole blood-based miRs in the diagnosis of intracranial aneurysm (IA) is still not clear. The present study aimed to examine miR expression profiling in the peripheral whole blood of IA patients and healthy controls.

Material and methods: Seventy-three IA patients, including 34 unruptured and 39 ruptured, and 28 healthy subjects, were recruited for diagnostic analysis. microRNA (miR) expression profiling in whole blood from healthy controls and IA patients was evaluated using miRNA microarray assay. RT-qPCR was used to evaluate miR expression. Receiver operating characteristics (ROC) curves and the area under the ROC curves (AUC) were used to calculate the diagnostic power of miRs in whole blood of IA.

Results: We observed significantly higher miR-21 and miR-92 expression levels in aneurysmal tissues and whole blood of IA patients as compared to healthy subjects. miR-21 expression level was significantly positively correlated with miR-92 in IA tissues and whole blood of IA patients. ROC analysis revealed that miR-21 (AUC $=0.843$, sensitivity $=0.849$, specificity $=0.750$ ) and miR-92 (AUC $=0.892$, sensitivity $=0.945$, specificity $=0.786$ ) were promising in diagnosis of IA with high detectability. Intriguingly, miR-21 combined with miR-92 markedly improved the diagnostic power of IA (AUC $=0.920$, sensitivity $=1.000$, specificity $=0.786)$.

Conclusions: miR-21 combined with miR-92 could be considered as a potential biomarker for IA screening.

Key words: miR-21, miR-92, whole blood, intracranial aneurysm, biomarker, diagnosis.

\section{Introduction}

Intracranial aneurysm (IA) is a cerebrovascular lesion characterized by aberrant dilatation of the cerebral artery [1]. The morbidity of IA is approximately $3 \%$ of the general population worldwide, about $2 \%$ of which will develop into ruptured IA and cause subarachnoid hemorrhage, accounting for approximately $10 \%$ of acute cerebral stroke cases [2, 3]. At present, the initiation and progression of IA formation and rupture are complicated. Even more unfortunately, in spite of the advances in techniques of surgical and interventional therapy, the mortality rate and undesirable prognosis in IA patients have failed to improve in clinical practice [3].

\author{
Corresponding author: \\ Dr. Hang Shu \\ Department \\ of Neurosurgery \\ Guangdong Provincial \\ People's Hospital \\ Guangdong Academy \\ of Medical Sciences \\ 106 Zhongshan Second Road \\ Guangzhou 510080 \\ Guangdong Province, China \\ Phone: +86 020-83827812 \\ Fax: +86 020-83827812 \\ E-mail: shu_hang11@163. \\ com
}


Some investigators have attempted to identify the differentially expressed genes between normal superficial temporal arteries (STA) and unruptured or ruptured IA tissues using microarray and polymerase chain reaction (PCR) techniques, suggesting that matrix metalloproteinase-13 (MMP-13) and collagen genes are up-regulated in IA tissues [4]. However, marker in IA tissue as the invasive diagnostic mode limits into routine clinical practice. More recently, there have been a growing number of publications focusing on blood-based non-invasive detection [2, 5], suggesting that numerous microRNAs (miRs) are differentially expressed in peripheral blood of IA patients and can serve as novel biomarkers for the diagnosis of IA.

MiRs as a class of non-coding RNAs perform a fundamental role to regulate gene expression via post-transcriptional repression [6, 7]. Numerous studies have confirmed that miRs participate in a variety of physiological and pathological processes, including cerebrovascular diseases [8, 9]. For example, miR-143, miR-145 and miR-448-3p are implicated in the pathogenesis of IA $[10,11]$ Down-regulation of miR-143 and miR-145 in plasma may be associated with IA formation and rupture [12]. Moreover, miRs have proved to be valuable diagnostic markers in various diseases [13, 14]. Some studies also suggest that the underlying mechanism for the alterations in peripheral blood miRs may be associated with the excessive release of miR from the nidus into the circulation $[15,16]$. In contrast, all of the cells from the body, not just cells in the nidus, can release miRs into the blood circulation, and nidus-released miRs are not enough to change the miR levels in the blood circulation, because the nidus constitutes only a very small fraction of the body's cellular mass [17]. Indeed, the changes of miR expression in serum, plasma and whole blood may be a systemic response in the body in response to disease conditions, including deregulated miRs in circulating blood cells $[18,19]$.

In our study, miRs expression profiling in peripheral whole blood of IA patients was explored using miR microarray assay. Two representative miRs (miR-21 and miR-92) in peripheral whole blood were selected through a comprehensive miRNA array-based approach. Furthermore, we confirmed that up-regulation of miR-21 and miR-92 in peripheral whole blood could serve as non-invasive biomarkers for IA screening.

\section{Material and methods}

\section{Patients and specimens}

Seventy-three pairs of Al and normal superficial temporal artery (STA) specimens were obtained from 73 IA patients, who underwent microsurgical clipping as described previously [4], from January 2011 to December 2016 at the Guangdong General Hospital (Guangzhou, China). Moreover, peripheral whole blood samples $(10 \mathrm{ml})$ were acquired from 28 healthy controls, seventy-three pre-operative and post-operative IA patients, and stored in an ultra-low temperature refrigerator (Thermo Fisher Scientific). Informed consent forms were collected from all participants. The Ethics Committee of the Guangdong Provincial People's Hospital, Guangdong Academy of Medical Sciences, approved this diagnostic study on January $10^{\text {th }}, 2011$ (approval number: 201101023).

\section{RNA isolation and reverse transcription- quantitative polymerase chain reaction (RT-qPCR)}

miRNeasy Mini Kit (Qiagen, Inc., Valencia, CA, USA) was used to extract total RNA, according to the manufacturer's protocol. TaqMan RT kit and TaqMan MicroRNA assay (Thermo Fisher Scientific, Inc.) were used to perform RT-qPCR assay of miRs, according to the manufacturer's protocol. miRs expression levels were calculated using the $\Delta C t$ method, as previously described [20].

\section{Microarray assays}

Microarray analysis was performed using Agilent Human miRNA (8*15K) V14.0 arrays at Ribobio (Guangzhou, China), as described previously [21].

\section{Statistical analysis}

Data were presented as the mean \pm standard deviation for each group. All statistical analyses were performed using SPSS version 17.0 (SPSS, Inc., Chicago, IL, USA) as described previously [21]. A $p$-value less than 0.05 was considered to indicate a statistically significant difference.

\section{Results}

\section{Clinical characteristics of healthy controls and IA cases}

In our study, 28 healthy controls and seventy-three IA cases, including 34 unruptured and 39 ruptured cases, were recruited for the marker diagnostic study. The systolic blood pressure in both unruptured and ruptured cases was significantly higher than that of the subjects in the healthy control group. Others parameters, including age, gender, diastolic blood pressure, history of hypertension, history of smoking and drinking and fasting blood glucose, had no obvious difference between the two groups. Moreover, we summarized the location of the diagnosed IA as shown in Table I. 
Table I. Clinical characteristics of healthy controls and patients with IA

\begin{tabular}{|c|c|c|c|}
\hline Paramtere & $\mathrm{HC}(n=28)$ & Unruptured $(n=34)$ & Ruptured $(n=39)$ \\
\hline Age [years] & $51.89 \pm 5.18$ & $52.62 \pm 5.38$ & $51.21 \pm 5.67$ \\
\hline Gender (male/female) & $17 / 11$ & $20 / 14$ & $23 / 16$ \\
\hline Systolic blood pressure [mm Hg] & $116 \pm 10$ & $123 \pm 15^{*}$ & $128 \pm 17^{\star *}$ \\
\hline Diastolic blood pressure [mm Hg] & $72 \pm 7$ & $76 \pm 9$ & $80 \pm 10^{*}$ \\
\hline History of hypertension & $15(53.6 \%)$ & $18(52.9 \%)$ & $21(53.8 \%)$ \\
\hline History of smoking and drinking & $12(42.8 \%)$ & $20(58.8 \%)$ & $24(61.5 \%)$ \\
\hline Fasting blood glucose [mmol/l] & $5.52 \pm 1.23$ & $5.67 \pm 1.45$ & $5.89 \pm 1.37$ \\
\hline \multicolumn{4}{|l|}{ Location: } \\
\hline Anterior communicating artery & / & 7 & 10 \\
\hline Posterior communicating artery & / & 4 & 3 \\
\hline Anterior cerebral artery & / & 6 & 3 \\
\hline Middle cerebral artery & / & 2 & 4 \\
\hline Posterior cerebral artery & / & 3 & 6 \\
\hline Internal carotid artery & / & 8 & 12 \\
\hline Vertebra artery & / & 4 & 1 \\
\hline
\end{tabular}

IA - intracranial aneurysm, HC - healthy controls. ${ }^{*} P<0.05,{ }^{* *} p<0.01$ vs. healthy control, 1-way ANOVA.

\section{miR expression profiling in whole blood} from healthy controls and IA patients

miR microarray assays were performed in nine whole blood samples, which were randomly selected from healthy controls, unruptured or ruptured IA cases ( $n=3$ in each group). Differentially expressed miRs were filtered based on fold change $\geq 2$, $p<0.001$ and FDR $<0.001$. Compared to healthy controls, 30 miRs were deregulated in whole blood from unruptured IA cases, among which 19 miRs were significantly up-regulated, and $11 \mathrm{miRs}$ were significantly down-regulated (Figure 1 A). In addition, we found that 39 miRs were changed (24 up-regulated and 15 down-regulated) in whole blood from ruptured IA cases compared with the healthy control group (Figure 1 B). Importantly, we found that 5 miRs (miR-9, miR-17, miR-21, miR-92 and miR-185) were simultaneously increased in both unruptured and ruptured IA cases, among which the fold change of miR-21 and miR-92 was obviously higher than miR-9, miR-17 and miR-185 (Figure $1 \mathrm{C}$ ). Therefore, we focused on miR-21 and miR-92 in the further experiments.

\section{Validation of miR-21 and miR-92 expression by RT-qPCR}

To investigate whether miR-21 and miR-92 could function as markers for IA diagnosis, we measured the expression of miR-21 and miR-92 in whole blood from $73 \mathrm{IA}$ cases and 28 healthy controls, as well as in 73 IA tissues and corre- sponding normal STA. The results demonstrated that miR-21 and miR-92 were significantly higher in IA patients, including whole blood and IA tissues, as compared to the corresponding control group (Figures $2 \mathrm{~A}, \mathrm{C}$ ). However, the expression of miR-21 and miR-92 in whole blood or IA tissues had no statistically significant difference between unruptured and ruptured IA cases (Figures 2 B, D).

\section{Comparison of miR-21 and miR-92 between whole blood and IA tissues}

To determine whether miR-21 and miR-92 served as blood-based non-invasive markers for IA, we performed a correlation analysis of miR-21 and miR-92 levels between whole blood and IA tissues. As shown in Figures $3 \mathrm{~A}$ and $\mathrm{B}$, the expression of miR-21 between whole blood and IA tissues was significantly and positively correlated in seventy-three IA cases $(r=0.672, p<0.001)$, and Bland-Altman analysis showed a high consistency of miR-21 expression in whole blood and IA tissues (mean differences $=-1.079 \pm 0.884 ; 95 \%$ limits of agreement: $-2.811,0.623)$. We also found that whole blood and IA tissues were strongly correlated for miR-92 expression $(r=0.634, p<0.001)$, and Bland-Altman analysis revealed a high concordance of miR-21 expression in whole blood and IA tissues (mean differences $=-1.138 \pm 0.806 ; 95 \%$ limits of agreement: $-2.718,0.442$ ) (Figures 3 C, D). Our results indicated that miR-21 and miR-92 in whole blood accurately reflected the actual yield 
A

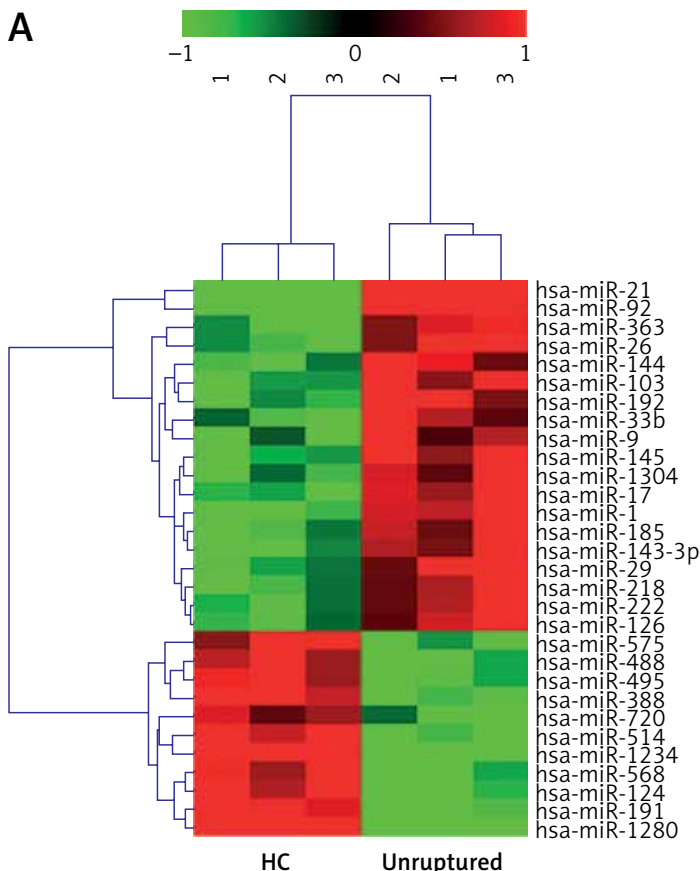

B

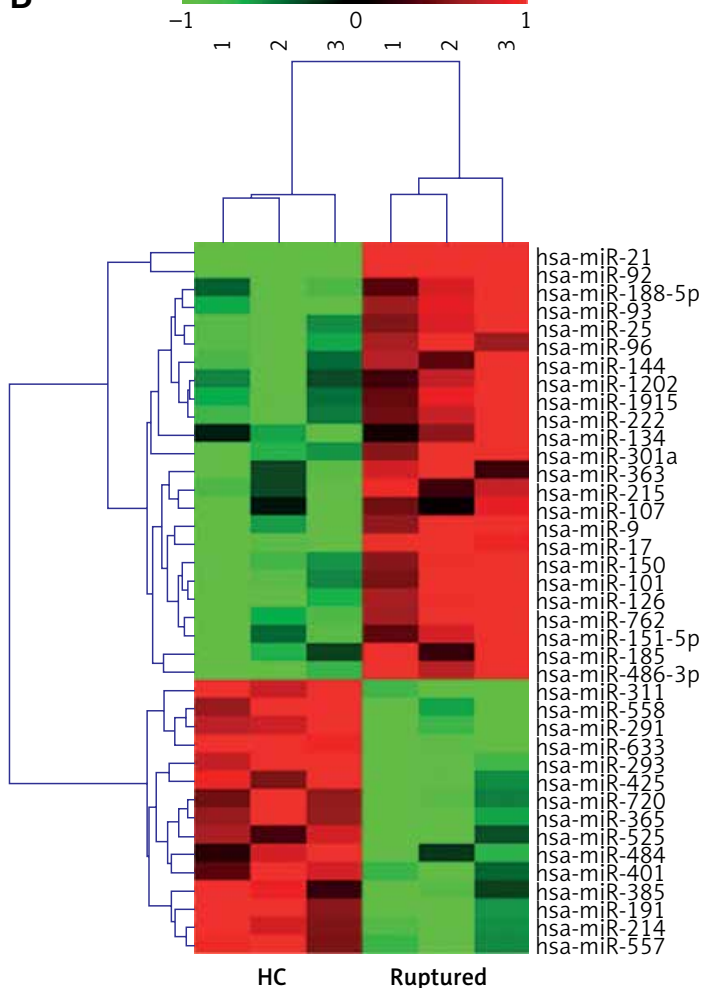

C

\begin{tabular}{|c|c|c|c|c|}
\hline \multirow[t]{2}{*}{ Variable } & \multicolumn{2}{|c|}{ Unruptured vs. HC } & \multicolumn{2}{|c|}{ Ruptured vs. HC } \\
\hline & Fold change & $P$-value & Fold change & $P$-value \\
\hline Hsa-miR-9 & 3.03 & $6.23 \times 10^{-4}$ & 4.08 & $1.82 \times 10^{-5}$ \\
\hline Hsa-miR-17 & 3.22 & $1.88 \times 10^{-3}$ & 4.76 & $2.56 \times 10^{-4}$ \\
\hline Hsa-miR-21 & 13 & $3.16 \times 10^{-5}$ & 12.64 & $1.04 \times 10^{-5}$ \\
\hline Hsa-miR-92 & 19.7 & $2.67 \times 10^{-4}$ & 20.11 & $8.27 \times 10^{-5}$ \\
\hline Hsa-miR-185 & 3.1 & $6.98 \times 10^{-3}$ & 3.16 & $1.96 \times 10^{-4}$ \\
\hline
\end{tabular}

Figure 1. miR expression profiling in whole blood from healthy controls and IA patients. miR microarray assays were used to measure differentially expressed miRs in whole blood from unruptured (A) or ruptured (B) IA compared with healthy controls $(\mathrm{HC})$ group. Each column represents an individual whole blood sample, and each row represents an individual miR. The color legend at the top of the heat map represents the expression levels of miR, red indicating high expression and green indicating low expression. $\mathbf{C}-$ miR microarray assays showed that 5 miRs were up-regulated in both unruptured and ruptured IA compared with $\mathrm{HC}$ group

of miRs in IA tissues, suggesting that both miR-21 and miR-92 were acceptable as blood-based markers for IA screening.

\section{Evaluation of stabilization of miR-21 and miR-92}

To investigate whether protracted processing of whole blood samples altered the expression of miR21 and miR-92, we kept the whole blood samples at room temperature for different times. The results demonstrated that the expression of miR-21 and miR-92 had no obvious change at room temperature within $18 \mathrm{~h}$, while the expression levels of miR-21 and miR-92 were significantly lower at $24 \mathrm{~h}$ as com- pared to $0 \mathrm{~h}$ (Figures $4 \mathrm{~A}, \mathrm{~B}$ ). These results showed a relatively dilatory degradation rate, which provided a high accuracy of analytical results of blood-based miR-21 and miR-92 levels for IA diagnosis.

\section{Comparison of miR-21 and miR-92 in pre-operation and post-operation}

To identify whether miR-21 and miR-92 were released from IA tissues into the circulation, we detected the levels of miR-21 and miR-92 in whole blood before and after the surgical operation. We found that whole blood levels of miR-21 and miR92 were significantly decreased 14 days after the surgical operation as compared to before the 
A

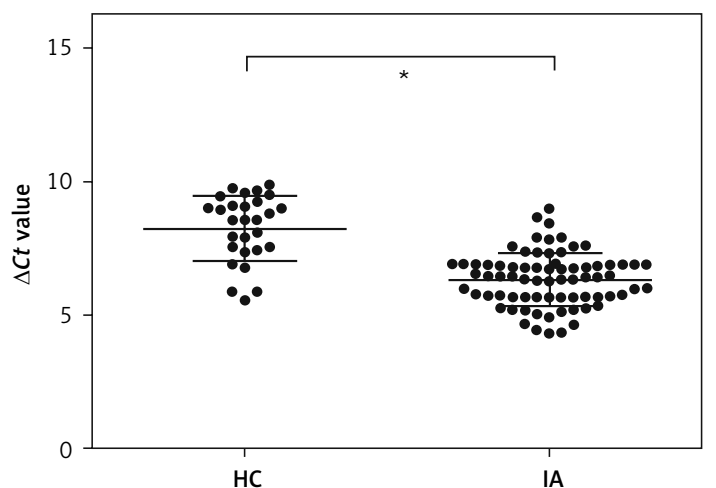

B

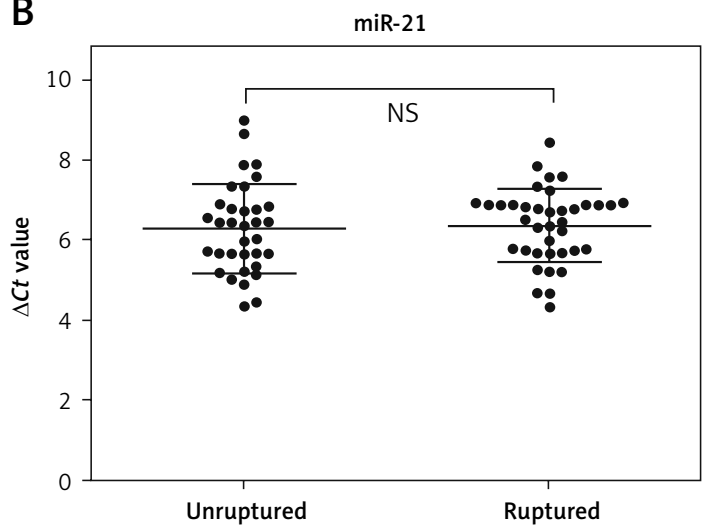

C

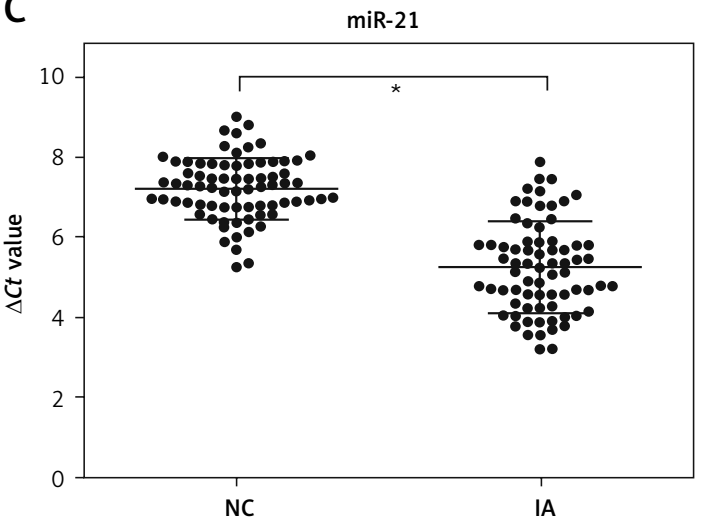

D



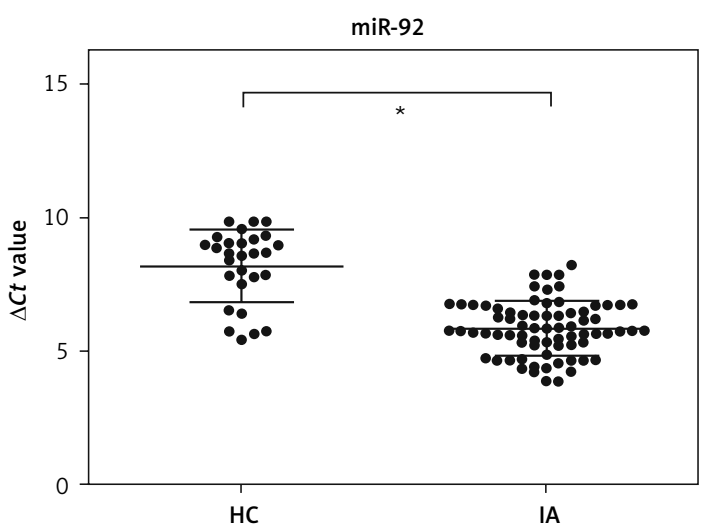

$\operatorname{miR-92}$
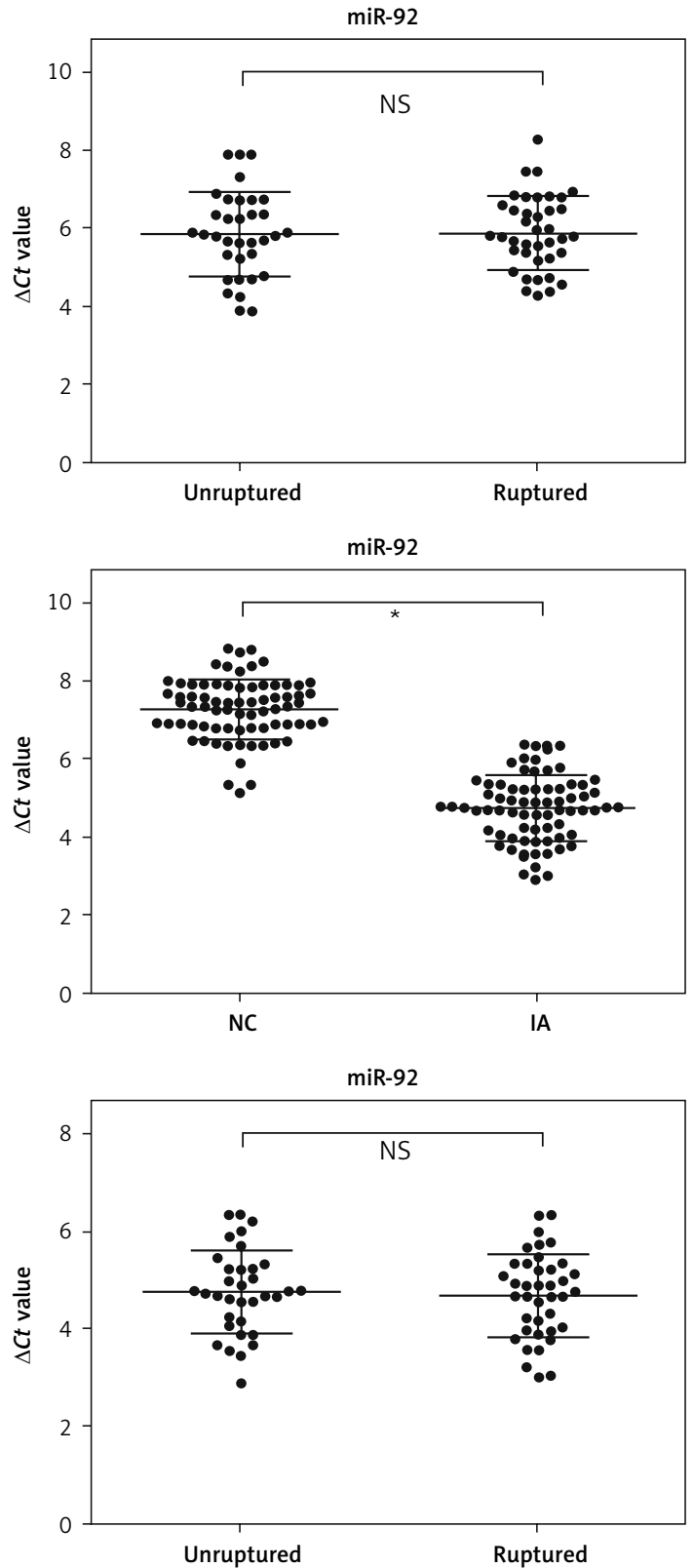

Figure 2. Expression of miR-21 and miR-92 was increased in both whole blood and aneurysmal tissues from IA patients. A comparison of miR-21 and miR-92 expression in whole blood was performed between healthy control and IA patients (A), as well as unruptured and ruptured IA (B). A comparison of miR-21 and miR-92 expression was performed between normal STA and IA tissues (C), as well as unruptured and ruptured aneurysmal tissues (D) ${ }^{*} P<0.05 ; n$. $\mathrm{s}$ represents no statistical significance. 
A

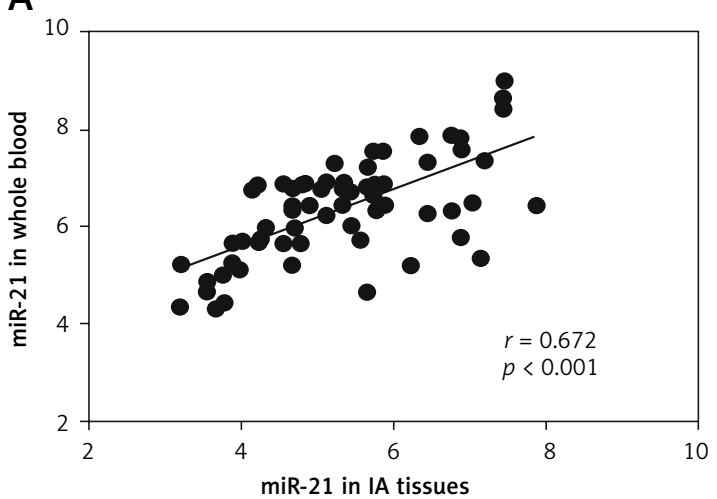

C

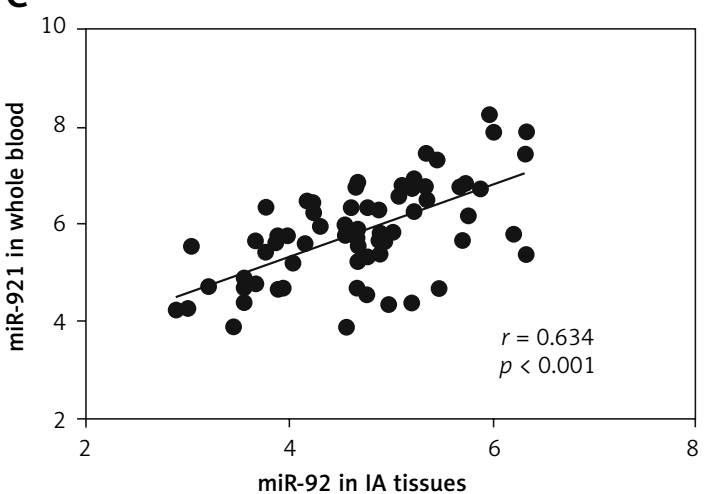

B



D

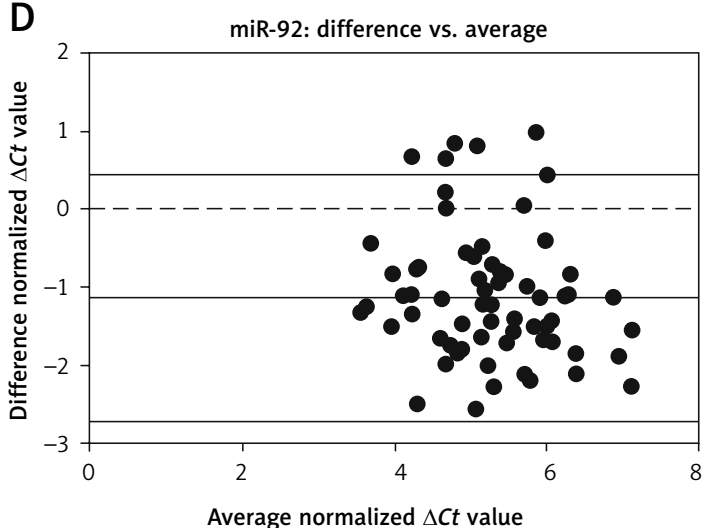

Figure 3. Correlation of miR-21 and miR-92 between whole blood and aneurysmal tissues was investigated. Spearman's rank analysis and Bland-Altman were used to measure the correlation analysis and consistency check of miR-21 (A, B) and miR-92 (C, D) expression between whole blood and aneurysmal tissues

A

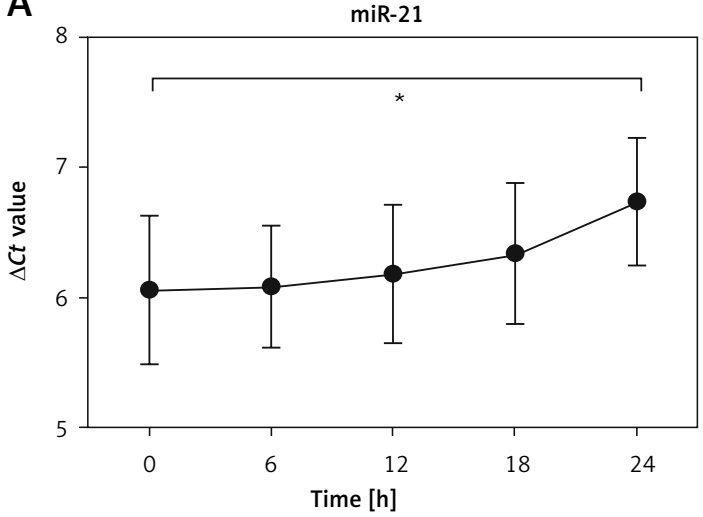

B



Figure 4. Evaluation of the stabilization of miR-21 and miR-92. Whole blood samples were stored at room temperature for different times; the levels of miR-21 (A) and miR-92 (B) were measured by RT-qPCR

${ }^{\star} P<0.05$.

surgical operation in IA patients (Figures $5 \mathrm{~A}, \mathrm{~B}$ ). These results suggested that the up-regulation of miR-21 and miR-92 levels in whole blood might be associated with the growth of IA tissues.

\section{Evaluation of miR-21 and miR-92 for IA diagnosis}

To investigate whether miR-21 and miR-92 could serve as blood-based markers for IA diag- nosis, ROC and AUC were performed to evaluate the clinical diagnostic significance of miR-21 and miR-92 in IA patients. The AUC of miR-21 and miR92 ROC was 0.843 (95\% confidence interval $(\mathrm{Cl})$ : $0.749-0.937)$ and 0.892 (95\% Cl: 0.814-0.971) at a diagnostic threshold of 7.33 and 7.51 , respectively (Table II, Figures 6 A, B). The sensitivity and specificity of miR-21 and miR-92 for IA diagnosis were 0.849 and 0.750 , and 0.945 and 0.786 , respectively (Table II, Figures 6 A, B). Furthermore, 
A

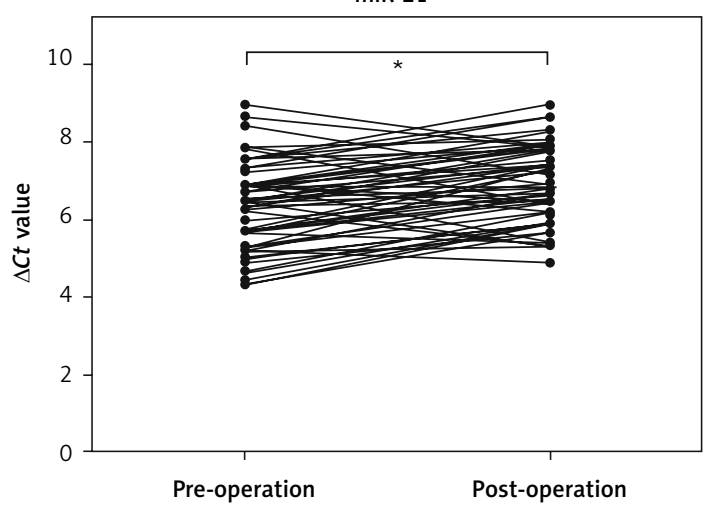

B

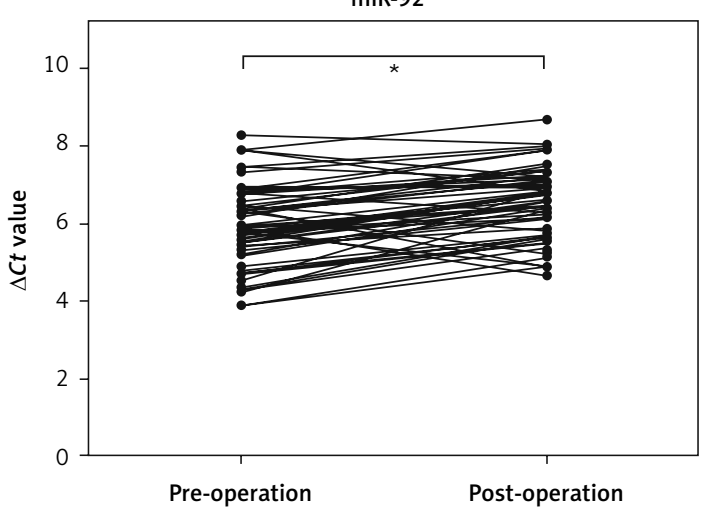

Figure 5. Comparison of miR-21 and miR-92 in pre-operation and post-operation. Seventy-three pairs of whole blood were acquired from IA patients before and after the surgical operation; the levels of miR-21 (A) and miR-92 (B) in whole blood were measured by RT-qPCR

${ }^{*} P<0.05$.

the ROC analysis was performed in the combination of miR-21 and miR-92. The AUC of the miR-21 combined with miR-92 ROC was $0.920(95 \% \mathrm{Cl}$ : $0.850-0.988)$, with sensitivity of 1.000 and specificity of 0.786 at a diagnostic threshold of 18.69 (Table II, Figure $6 \mathrm{C}$ ).

\section{Discussion}

Recently, differentially expressed miRs in whole blood have been detected in both non-malignant conditions, such as type 2 diabetes mellitus [22], rheumatoid arthritis [23] and acute myocardial infarction [24], and cancer conditions, such as liposarcoma [25], lung adenocarcinoma [13] and prostate cancer [26]. Therefore, there have been a growing number of publications focusing on blood-based biomarkers for clinical diagnosis [26]. In the process of IA, numerous miRs are deregulated in aneurysmal tissue and plasma samples and can serve as new biomarkers for IA diagnosis $[2,14,27]$. In the present study, we have renewed interest in whole blood-based miRs for IA diagnosis. IA-related miRs screening was performed in peripheral whole blood based on different expression profiling between healthy controls and IA patients using miR microarray assays. The results demonstrated that up-regulation of miR-21 and miR-92 was identified in whole blood and aneurysmal tissue from IA patients, and then further measurements showed that miR-21 combined with miR-92 exhibited the highest diagnostic power for the diagnosis of IA (AUC $=0.920$, sensitivity: 1.000 and specificity: 0.786), indicating that miR-21 combined with miR-92 could be used as combined diagnostic markers for IA.

Previous studies have demonstrated that an increase in levels of immunologic markers, inflammatory cytokines, cell adhesion molecules and enzymes, including complement C3 and C9, interleukin-1 $\beta$ and tumor necrosis factor $\alpha$ (TNF- $\alpha$ ), intercellular adhesion molecule 1 (ICAM-1) and vascular cell adhesion molecule 1 (VCAM), angiotensin convertase enzyme (ACE) and elastase-to- $\alpha 1$-antitrypsin are seen in aneurysm tissue and blood $[1,28]$. It is important to note that these molecules are closely related to brain and vascular damage and aneurysm rupture [1, 29], suggesting that IA development and progression to rupture may be influenced by multiple factors. It is generally believed that complicated pathogenesis of IA leads to a huge challenge of the early detection of IA. Although radiographic imaging of the cerebral vasculature is proved as the only method for aneurysm detection, the asymptomatic clinical presentation and high expense of IA patients reduce the diagnostic rate of IA. Interestingly, biomarkers for noninvasive screening are emerging as effective methods for IA screening [2, 4, 27]. For

Table II. Performance of miR-21 and miR-92 in differential diagnosis of IA from healthy controls

\begin{tabular}{|c|c|c|c|c|c|c|c|c|}
\hline \multirow[t]{2}{*}{ Variable } & \multirow[t]{2}{*}{ AUC } & \multirow[t]{2}{*}{$P$-value } & \multicolumn{2}{|c|}{$95 \% \mathrm{Cl}$} & \multirow[t]{2}{*}{ Sensitivity } & \multirow[t]{2}{*}{ Specificity } & \multirow{2}{*}{$\begin{array}{l}\text { Youden } \\
\text { index }\end{array}$} & \multirow{2}{*}{$\begin{array}{c}\text { Cut off } \\
\text { point }\end{array}$} \\
\hline & & & Lower & Upper & & & & \\
\hline miR-21 & 0.843 & $<0.001$ & 0.749 & 0.937 & 0.849 & 0.750 & 0.599 & 7.33 \\
\hline miR-92 & 0.892 & $<0.001$ & 0.814 & 0.971 & 0.945 & 0.786 & 0.731 & 7.51 \\
\hline $\begin{array}{l}\text { miR-21 + } \\
\text { miR-92 }\end{array}$ & 0.920 & $<0.001$ & 0.850 & 0.988 & 1.000 & 0.786 & 0.786 & $18.69^{\circ}$ \\
\hline
\end{tabular}

IA - intracranial aneurysm, AUC - area under receiver operating characteristics curves, $\mathrm{Cl}$ - confidence interval. 

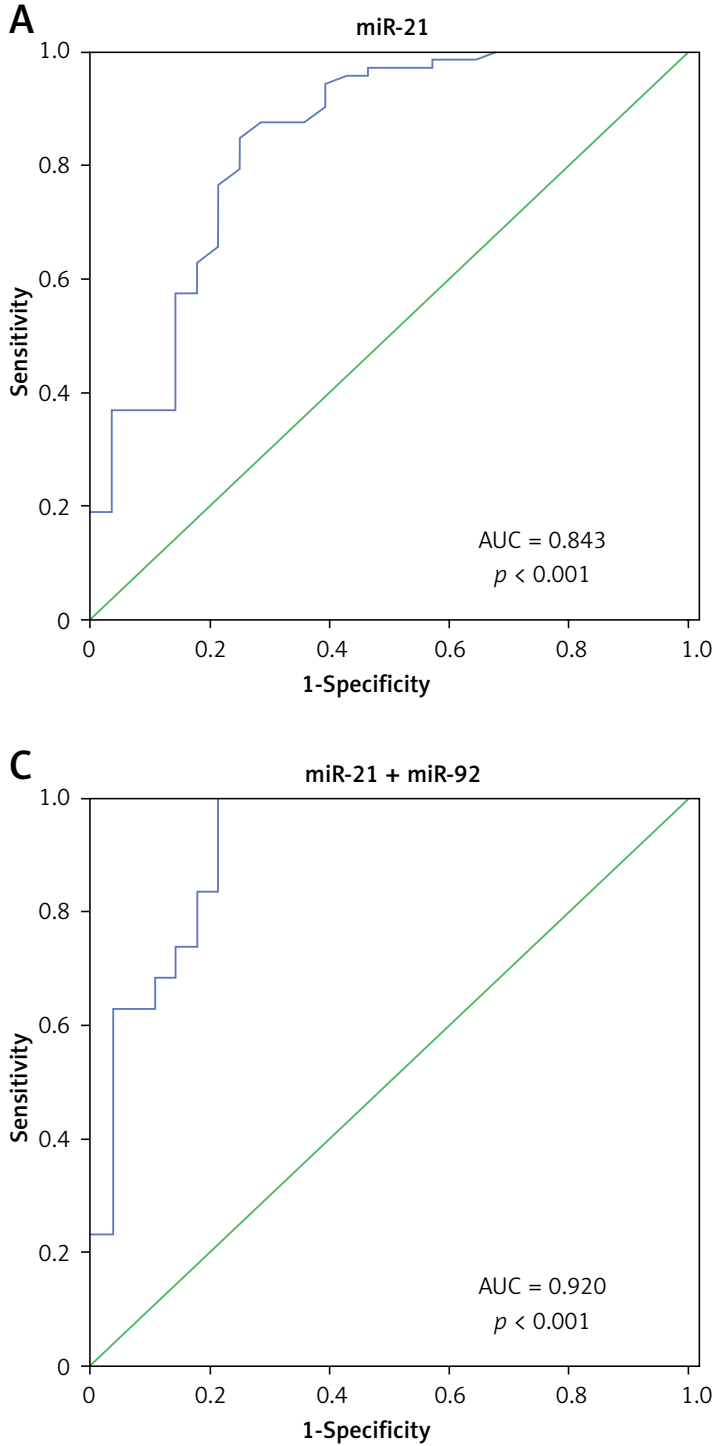

example, miR-29a is increased in the plasma from IA patients and possesses a high effectiveness in IA diagnosis [27]. A comprehensive analysis of miR expression profiling in plasma from IA patients indicates that 99 miRs are specifically changed in IA patients, suggesting that blood levels of miRs may be useful as a simple and rapid alternative measure for IA screening [2]. In our study, we found that five miRs (miR-9, miR-17, miR-21, miR-92 and miR-185) were up-regulated and participated in IA formation and progression to rupture. Further experiments showed that miR-21 and miR-92 could be used as a diagnostic tool for screening IA patients from apparently healthy individuals. More importantly, a combination of miR-21 and miR-92 for IA screening showed a higher AUC, sensitivity and specificity than miR-21 or miR-92 alone. The expression levels of miR-21 and miR-92 in whole blood were also compared in two subgroups: the unruptured and ruptured aneurysm groups. However, the expression levels of miR-21 and miR-92

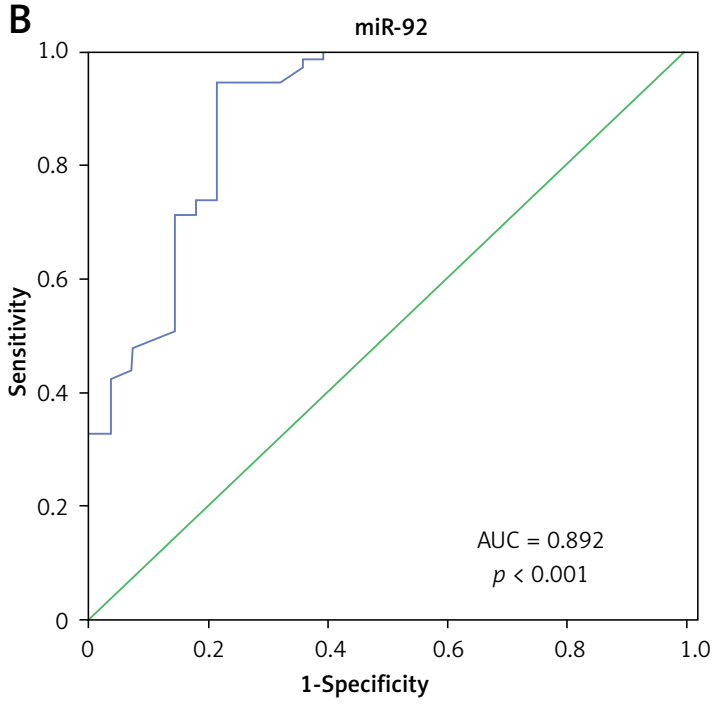

Figure 6. Evaluation of miR-21 and miR-92 for IA diagnosis. Receiver operating characteristics (ROC) curves were drawn with the data of whole blood miR-21 (A) and miR-92 (B) levels from 73 IA patients and 28 healthy controls. Using binary logistic regression analysis, the ROC curves were drawn with the data of whole blood miR-21 combined with miR-92 for IA diagnosis (C)

showed no significant difference between the two groups. Therefore, whether miR-21 and miR-92 could be indicators for predicting the rupture risk of IA has not been investigated.

Conventional wisdom may suggest that circulating RNAs are unstable because of the high level of RNase activity in plasma [30]. However, Tong et al. revealed that long non-coding RNAs (IncRNAs) are remarkably stable in plasma from cancer patients and can resist RNase A digestion, multiple freeze-thaw cycles and strong acid treatment. The underlining mechanism is that IncRNAs can form relatively stable secondary structures [20]. In the present study, we found that circulating miRs were stable in peripheral whole blood at room temperature within 18 hours, which provided plenty of time for clinical examination.

We were surprised to find that miR-21 and miR-92 were significantly lower in whole blood of patients with a surgical operation than before the surgical operation. This result may support 
the hypothesis that RNAs in blood associate with the microenvironments of pathological tissues [31]. On the other hand, some researchers suggest that blood cells were the major contributor to the up-regulation of RNA in pathologic conditions [32]. Inflammation as a main risk factor accelerates IA development and progression to rupture [33]. A systematic or local inflammatory response can promote miR expression in aneurysmal tissues and whole blood [14, 34]. At present, it is difficult to explain the deregulation of miRs in whole blood, while most researchers consider that blood cells and the metabolism of lesion tissues have come together to regulate miR expression in whole blood [13, 17].

However, there are some limitations in our study: (1) the number of samples was limited, which might lead to the systematic bias of measurement results; (2): the precise mechanism of circulating miRs resistant to endogenous RNase digestion has not been represented in the present study; (3) the secrete mode of whole blood miRs in IA patients did not involve.

In conclusion, the present results demonstrate that miR-21 combined with miR-92 could function as a potential biomarker for IA screening.

\section{Conflict of interest}

The authors declare no conflict of interest.

\section{References}

1. Hussain S, Barbarite E, Chaudhry NS, et al. Search for biomarkers of intracranial aneurysms: a systematic review. World Neurosurg 2015; 84: 1473-83.

2. Li P, Zhang Q, Wu X, et al. Circulating microRNAs serve as novel biological markers for intracranial aneurysms. J Am Heart Assoc 2014; 3: e000972.

3. Santiago-Sim T, Fang X, Hennessy ML, et al. THSD1 (thrombospondin type 1 domain containing protein 1) mutation in the pathogenesis of intracranial aneurysm and subarachnoid hemorrhage. Stroke 2016; 47: 3005-13.

4. Bekelis K, Kerley-Hamilton JS, Teegarden A, et al. MicroRNA and gene expression changes in unruptured human cerebral aneurysms. J Neurosurg 2016; 125: 1390-9.

5. Bo L, Wei B, Wang Z, Kong D, Gao Z, Miao Z. Screening of critical genes and microRNAs in blood samples of patients with ruptured intracranial aneurysms by bioinformatic analysis of gene expression data. Med Sci Monit 2017; 23: 4518-25.

6. Vidigal JA, Ventura A. The biological functions of miRNAs: lessons from in vivo studies. Trends Cell Biol 2015; 25: 137-47.

7. Li J, Wu G, Cao Y, Hou Z. Roles of miR-210 in the pathogenesis of pre-eclampsia. Arch Med Sci 2019; 15: 183-90.

8. Lopes KP, Vinasco-Sandoval T. Global miRNA expression profile reveals novel molecular players in aneurysmal subarachnoid haemorrhage. Sci Rep 2018; 8: 8786.

9. Sheng B, Fang X, Liu C, et al. Persistent high levels of miR-502-5p are associated with poor neurologic outcome in patients with aneurysmal subarachnoid hemorrhage. World Neurosurg 2018; 116: e92-9.
10. Zhang JZ, Chen D, Lv LQ, et al. miR-448-3p controls intracranial aneurysm by regulating KLF5 expression. Biochem Biophys Res Commun 2018; 505: 1211-5.

11. Xu J, Yan S, Tan H, et al. The miR-143/145 cluster reverses the regulation effect of KLF5 in smooth muscle cells with proliferation and contractility in intracranial aneurysm. Gene 2018; 679: 266-73.

12. Feng X, Peng F, Zhang B, et al. Lower miR-143/145 and higher matrix metalloproteinase-9 levels in circulation may be associated with intracranial aneurysm formation and rupture: a pilot study. Clin Neurol Neurosurg 2018; 173: 124-9.

13. Patnaik SK, Yendamuri S, Kannisto E, Kucharczuk JC, Singhal S, Vachani A. MicroRNA expression profiles of whole blood in lung adenocarcinoma. PLoS One 2012; 7: e46045.

14. Liu D, Han L, Wu X, Yang X, Zhang Q, Jiang F. Genome-wide microRNA changes in human intracranial aneurysms. BMC Neurol 2014; 14: 188.

15. Mitchell PS, Parkin RK, Kroh EM, et al. Circulating microRNAs as stable blood-based markers for cancer detection. Proc Natl Acad Sci USA 2008; 105: 10513-8.

16. Brase JC, Johannes M, Schlomm T, et al. Circulating miRNAs are correlated with tumor progression in prostate cancer. Int J Cancer 2011; 128: 608-16.

17. Chen X, Liang H, Zhang J, Zen K, Zhang CY. Secreted microRNAs: a new form of intercellular communication. Trends Cell Biol 2012; 22: 125-32.

18. Silva AM, Almeida MI, Teixeira JH, et al. Profiling the circulating miRnome reveals a temporal regulation of the bone injury response. Theranostics 2018; 8: 3902-17.

19. Gupta P, Liu B, Wu JQ et al. Genome-wide mRNA and miRNA analysis of peripheral blood mononuclear cells (PBMC) reveals different miRNAs regulating $\mathrm{HIV} / \mathrm{HCV}$ co-infection. Virology 2014; 450-451: 336-49.

20. Tong YS, Wang XW, Zhou XL, et al. Identification of the long non-coding RNA POU3F3 in plasma as a novel biomarker for diagnosis of esophageal squamous cell carcinoma. Mol Cancer 2015; 14: 3.

21. Sun Y, Mei H, Xu C, Tang H, Wei W. Circulating microRNA-339-5p and - 21 in plasma as an early detection predictors of lung adenocarcinoma. Pathol Res Pract 2018; 214: 119-25.

22. Morais Junior GS, Souza VC, Machado-Silva W, et al. Acute strength training promotes responses in whole blood circulating levels of miR-146a among older adults with type 2 diabetes mellitus. Clin Interv Aging 2017; 12: 1443-50.

23. Mookherjee N, El-Gabalawy HS. High degree of correlation between whole blood and PBMC expression levels of miR-155 and miR-146a in healthy controls and rheumatoid arthritis patients. J Immunol Methods 2013; 400-401: 106-10.

24. Meder B, Keller A, Vogel B, et al. MicroRNA signatures in total peripheral blood as novel biomarkers for acute myocardial infarction. Basic Res Cardiol 2011; 106: 13-23.

25. Fricke A, Cimniak AFV, Ullrich PV, et al. Whole blood miRNA expression analysis reveals miR-3613-3p as a potential biomarker for dedifferentiated liposarcoma. Cancer Biomark 2018; 22: 199-207.

26. Pang C, Liu M, Fang W, et al. MiR-139-5p is increased in the peripheral blood of patients with prostate cancer. Cell Physiol Biochem 2016; 39: 1111-7.

27. Wang WH, Wang YH, Zheng LL, Li XW, Hao F, Guo D. MicroRNA-29a: a potential biomarker in the development of intracranial aneurysm. J Neurol Sci 2016; 364: 84-9.

28. Signorelli F, Sela S, Gesualdo L, et al. Hemodynamic stress, inflammation, and intracranial aneurysm devel- 
opment and rupture: a systematic review. World Neurosurg 2018; 115: 234-44.

29. Polin RS, Bavbek M, Shaffrey ME, et al. Detection of soluble E-selectin, ICAM-1, VCAM-1, and L-selectin in the cerebrospinal fluid of patients after subarachnoid hemorrhage. J Neurosurg 1998; 89: 559-67.

30. Tsui NB, Ng EK, Lo YM. Stability of endogenous and added RNA in blood specimens, serum, and plasma. Clin Chem 2002; 48: 1647-53.

31. Chen G, Wang J, Cui Q. Could circulating miRNAs contribute to cancer therapy? Trends Mol Med 2013; 19: 71-3.

32. Pritchard CC, Kroh E, Wood B, et al. Blood cell origin of circulating microRNAs: a cautionary note for cancer biomarker studies. Cancer Prev Res (Phila) 2012; 5: 492-7.

33. Tulamo R, Frosen J, Hernesniemi J, Niemela M. Inflammatory changes in the aneurysm wall: a review. J Neurointerv Surg 2018; 10: i58-67.

34. Li L, Sima X, Bai P, et al. Interactions of miR-34b/c and TP53 polymorphisms on the risk of intracranial aneurysm. Clin Dev Immunol 2012; 2012: 567586. 